\title{
Cuéntame la historia de tus abuelos: Ciencias Sociales, Matemáticas y Lengua en el grado de Educación Primaria
}

Recepción: 26/10/2020 | Revisión: 22/12/2020 | Aceptación: 09/08/2021 | Publicación: 01/03/2022

\author{
Queca CRIACH SINGLA \\ Blanquerna - Universitat Ramon Llull \\ quecacs@blanquerna.url.edu \\ http://orcid.org/oooo-0002-2562-3920
}

\author{
iD Montserrat PRAT MORATONAS \\ Blanquerna - Universitat Ramon Llull \\ montserratpm3@blanquerna.url.edu \\ https://orcid.org/oooo-0oo2-8979-7663 \\ Míriam TURRÓ AMORÓS \\ Blanquerna - Universitat Ramon Llull \\ miriamta1@blanquerna.url.edu \\ http://orcid.org/oooo-0003-0185-1555
}

\begin{abstract}
Resumen: A veces las oportunidades aparecen cuando los docentes compartimos lo que hacemos. Así empieza nuestra experiencia y la experiencia de nuestros alumnos. A principio del curso 2019-20 tres docentes que comparten un grupo de 2. ${ }^{\circ}$ curso del grado de Educación Primaria ven la oportunidad de ofrecer a los alumnos la conexión entre sus disciplinas, con la convicción de que en educación primaria las propuestas pueden ser un continuo y no deben presentarse como actividades desconectadas. Desde sociales se propone llegar al contenido curricular a través de objetos cotidianos de los abuelos de los alumnos (bottom-up). Desde matemáticas se realiza una línea del tiempo con dichos objetos, lo que permitirá reflexionar acerca de patrones y numeración. Desde lengua y literatura se construye una narración de la historia de los objetos situados en una línea temporal trabajando así, la estructura del texto narrativo. La experiencia culmina con la creación de distintas narrativas donde los estudiantes del grado de Educación Primaria conectan tres materias, experimentan cómo desarrollar una actividad interdisciplinar con toda su riqueza y nos hablan de las vidas de sus abuelos. En el transcurso de la experiencia irrumpe el confinamiento debido a la COVID-19, teniendo que adaptarla a la nueva situación.
\end{abstract}

Palabras clave: interdisciplinariedad; conocimiento del medio cultural y social; didáctica de la lengua y la literatura; didáctica de las matemáticas; COVID-19. 


\title{
TELL ME ABOUT THE LIVES OF YOUR GRANDPARENTS: SOCIAL SCIENCES, MATHEMATICS AND LANGUAGE IN A PRIMARY EDUCATION DEGREE
}

\begin{abstract}
Opportunities sometimes emerge when teachers share what they do. This was the origin of this experience. At the beginning of the 2019-2O academic year, three teachers who shared a second-year group pursuing a Primary Education degree spotted an opportunity to offer the students a link between their disciplines, with the conviction that in primary education the proposals should form a continuum and not be presented as disconnected activities. From the perspective of the social sciences, the use of everyday objects belonging to the students' grandparents was proposed to tackle the curricular content (bottom-up). In mathematics, these objects were situated on a timeline, which enabled the reflection on patterns and numbering. Through language and literature, the story of the objects was constructed, located along a timeline to work on the structure of the narrative text. The experience was culminated with the creation of different narratives where these Primary Education degree students linked up the three suject areas. They learnt how to develop an interdisciplinary activity in all its richness, and they were able to tell us about their grandparents' lives. The project was interrupted by COVID-19 and the ensuing confinement and it had to be adapted to the new situation.
\end{abstract}

Keywords: interdisciplinarity; social sciences; language and literature didactics; didactics of mathematics; COVID-19.

\section{EXPLICA'M LA HISTÒRIA DELS TEUS AVIS: CIÈNCIES SOCIALS, MATEMÀTIQUES I LLENGUA EN EL GRAU D'EDUCACIÓ PRIMÀRIA}

Resum: A vegades les oportunitats apareixen quan els docents compartim el que fem. Així comença la nostra experiència i l'experiència dels nostres alumnes. A principi del curs 2019-2020, tres docents que comparteixen un grup de $2 n$ curs del Grau d'Educació Primària veuen l'oportunitat d'oferir als alumnes la connexió entre les seves disciplines, amb la convicció que a educació primària les propostes poden ser un contínuum i no han de presentar-se com activitats desconnectades. Des de socials es proposa cobrir el contingut curricular a través d'objectes quotidians dels avis dels alumnes (bottom-up). Des de matemàtiques es realitza una línia del temps amb aquests objectes que permetrà reflexionar sobre patrons i numeració. Des de llengua i literatura es construeix una narració de la història dels objectes situats en una línia temporal, treballant així l'estructura del text narratiu. L'experiència culmina amb la creació de diverses narratives on els estudiants del grau d'Educació Primària connecten tres matèries, experimenten com desenvolupar una activitat interdisciplinar amb tota la seva riquesa $i$ ens parlen de les vides dels seus avis. En el transcurs de l'experiència irromp el confinament degut a la COVID-19, per la qual cosa es va haver d'adaptar l'experiència a la nova situació.

Paraules clau: interdisciplinarietat; coneixement del medi cultural i social; didàctica de la llengua i la literatura; didàctica de las matemàtiques; COVID-19.

\section{Introducción}

Las asignaturas en las facultades de Educación se organizan casi de forma exclusiva por disciplinas, mientras que en las aulas de primaria los maestros tienen el reto de conectarlas para ofrecer a los alumnos un aprendizaje significativo, globalizado, real, y conectado con el mundo que les rodea. Lo que puede parecer un contrasentido no lo es. Desde las facultades de 
Educación debemos fortalecer el conocimiento de las disciplinas y su didáctica para que los futuros maestros puedan identificarlo e incorporarlo correctamente en las diferentes actividades que propongan. Y, a su vez, como docentes del grado de Educación Primaria, debemos encontrar caminos para que los futuros maestros descubran y aprendan a conectar la realidad y las diferentes disciplinas.

La experiencia que se presenta a continuación parte del trabajo conjunto que tres docentes del grado de Educación Primaria llevaron a cabo durante el segundo semestre del curso 2019-20. Las tres profesoras, que compartían un mismo grupo de 45 alumnos, vieron la oportunidad de trabajar de manera interdisciplinaria las asignaturas que imparten: Actualización Curricular y Científica de las Ciencias Sociales; Didáctica de la Lengua y la Literatura y Didáctica de las Matemáticas. Mientras los alumnos cursaban las tres materias de manera paralela, una de las tareas que se les propuso fue un trabajo conjunto de estas asignaturas que debía permitirles conectar y construir conocimiento a partir de las tres disciplinas. El objetivo principal de la propuesta era que los alumnos experimentasen situaciones de aprendizaje similares a las que sus futuros alumnos vivirán, siendo una experiencia que buscaba potenciar el trabajo colaborativo en grupo y la integración de las tres disciplinas.

\section{La docencia compartida para trabajar interdisciplinariamente}

La experiencia que se presenta en este artículo se plantea desde la docencia compartida, con la certeza de que el trabajo conjunto enriquecerá el producto final. Partiendo de hechos y objetos reales se presenta una propuesta interdisciplinar que busca ir más allá de cada disciplina, pero sin perder la esencia de cada una. Por ello, consideramos interesante tener en cuenta algunas consideraciones sobre la docencia compartida y la interdisciplinariedad.

\subsection{Consideraciones sobre la docencia compartida}

La docencia compartida no tiene una única aproximación; la diversidad de los centros, de los equipos y de los niveles educativos multiplica sus posibilidades según las diferentes realidades. La codocencia, co-teaching, enseñanza colaborativa, enseñanza en equipo, o coenseñanza es un proceso formativo que llevan a cabo dos o más profesionales que colaboran para ofrecer de manera conjunta formación a un grupo de estudiantes, en un espacio físico determinado y con contenidos y objetivos específicos, con el fin de lograr lo que no podrían hacer solos (Suárez-Díaz, 2016, a partir de los trabajos de Cook y Friend, 1995; Cook, 2004; Beninghof, 2012). La coenseñanza permite a los docentes compartir y combinar sus habilidades y conocimientos con el objetivo de crear entornos de aprendizaje flexibles, rigurosos, basados en los currículums pero que a su vez se adapten a las necesidades de aprendizaje de los estudiantes (Friend, 2008).

Si ponemos el foco en si la docencia se desarrolla de manera simultánea o no, Dugan y Letterman (2008) describen tres tipos de docencia compartida: el co-teaching o docencia que tiene lugar cuando dos docentes enseñan de manera simultánea en un curso; el tipo alternate que se produce cuando dos docentes alternan la docencia; y en tercer lugar el team teaching que implica que haya un equipo docente que trabaja conjuntamente en la planificación pero donde cada miembro del equipo imparte una parte del curso. 
Otra aproximación relativa a los tipos de docencia compartida se centra en el grado de interacción entre los codocentes. Suárez-Díaz (2016) establece seis enfoques: uno enseña, uno observa; uno enseña, uno circula; enseñanza en paralelo; estaciones de enseñanza; enseñanza alternativa o diferenciada y equipo docente.

Sea cual sea la opción elegida de enseñanza compartida, es fundamental tener presente sus tres componentes: la co-planificación, la co-instrucción y la co-evaluación (Conderman y Hedin, 2012).

\subsection{Consideraciones sobre el concepto de interdisciplinariedad}

El concepto de interdisciplinariedad es esencial en el sí de la experiencia que aquí se presenta. Este término nace a mediados del siglo XX junto con el de pluridisciplinariedad. Aparece como respuesta a una indispensable necesidad de establecer puentes entre las diferentes disciplinas (Nicolescu, 1998). Para este autor, "Disciplinarity, multidisciplinarity, interdisciplinarity and transdisciplinarity are like four arrows shot from but a single bow: knowledge" (Nicolescu, 1998, p. 1).

En definitiva, los conceptos parten de las disciplinas, pero buscan establecer relaciones entre ellas con el fin de conseguir que el conocimiento avance (Folch et al., 2019). Las disciplinas, por lo tanto, son necesarias, pero no suficientes para abordar el conocimiento de una realidad compleja, siendo complementarias a las aproximaciones desde los ámbitos multi, trans o interdisciplinarios (Andonegui, 2004).

La revisión de la literatura permite observar que no existe una definición única del término interdisciplinariedad encontrándose, como señala Andonegui (2004), diversas interpretaciones, pero también niveles de rigor acerca de este concepto. De manera general podemos considerar la interdisciplina como "los modelos, leyes, categorías, técnicas, etc., provenientes de disciplinas científicas diferentes, que se mezclan entre sí para promover un conocimiento nuevo, un producto que resulte diferente de lo que existía en las disciplinas que contribuyeron a configurarlo" (Follari, 2013, p. 123). En definitiva, la interdisciplinariedad "designa las interacciones eficaces tejidas entre dos o más disciplinas y sus conceptos, sus procedimientos metodológicos, técnicas, etc. Por lo tanto, no es compatible con ninguna perspectiva acumulativa, porque impone interacciones reales" (Lenoir, 2013, p. 61).

Según Lenoir (2013), el término interdisciplinar es un concepto altamente polisémico; esto lleva a establecer cuatro campos donde puede operar: interdisciplinariedad práctica, interdisciplinariedad científica, interdisciplinariedad escolar y interdisciplinariedad profesional; cada una de ellas con sus particularidades y diferencias. Para este autor, la interdisciplinariedad escolar tiene como finalidad la difusión del saber científico a la vez que la formación de actores sociales, mediante la consecución de "condiciones adecuadas que permitan producir y apoyar el desarrollo de procesos integradores y la apropiación de saberes como productos cognitivos en los alumnos" (Lenoir, 2013, p. 65). Por consiguiente, es necesario ajustar los saberes escolares a nivel curricular, didáctico y pedagógico, siendo conveniente generar "vínculos de complementariedad entre las disciplinas escolares" (Lenoir, 2013, p. 65).

Al iniciar un trabajo interdisciplinar, es recomendable tener presentes los cinco atributos fundamentales de la interdisciplinariedad (Lenoir, 2013, pp. 67-73): 
- $\quad$ No existe la interdisciplinariedad sin disciplinas.

- La interdisciplinariedad no es la pluridisciplinariedad.

- $\quad$ La interdisciplinariedad requiere de tensión a nivel de finalidades.

- La interdisciplinariedad es un medio, la integración es la finalidad del proceso de aprendizaje.

- La interdisciplinariedad en la educación: una perspectiva relacional.

\section{Características de la experiencia: Nuestra maleta}

En este apartado se exponen las características generales de la experiencia. En primer lugar, se contextualiza; posteriormente, se presenta una descripción de la misma y, finalmente, se exponen los objetivos, contenidos y competencias a trabajar con esta propuesta.

\subsection{Contextualización y descripción de la experiencia}

La experiencia se llevó a cabo con estudiantes universitarios de segundo curso del grado de Educación Primaria de la Facultad de Psicología, Ciències de I'Educació i de l'Esport (FPCEE) Blanquerna de la Universitat Ramon Llull. Este proyecto nació de la inquietud de las tres docentes que, como se ha relatado anteriormente, comparten la convicción de la importancia y la riqueza del trabajo interdisciplinario.

A este hecho cabe sumar la coincidencia de que las tres asignaturas mencionadas se imparten a lo largo del segundo semestre del segundo año del grado de Educación Primaria en la FPCEE-Blanquerna. Las tres implican una importante carga didáctica en la concepción de las mismas. Esta situación facilitó enormemente la puesta en marcha de la experiencia conjunta que enriqueció y retroalimentó no solamente los planteamientos didácticos de cada asignatura, sino y sobre todo, los que afectaban a las tres en su conjunto.

En este período lectivo irrumpió la pandemia del virus del COVID-19 y ello conllevó que las sesiones presenciales se vieran interrumpidas, con lo cual fue necesario repensar la planificación y la organización de las diversas acciones y actividades que se habían pensado inicialmente. No obstante, las competencias, los objetivos, y los contenidos que se tuvieron en cuenta desde cada materia, pero también los compartidos por las tres asignaturas y que se detallan a continuación, no se vieron modificados a pesar de la situación de confinamiento.

En la experiencia que se propuso, los alumnos se distribuyeron en ocho grupos heterogéneos de cinco o seis participantes organizados cooperativamente. Construyeron un texto final multimodal que narraba una historia a partir del conjunto de objetos que previamente los estudiantes seleccionaron y que transitan a través de momentos históricos concretos. Así, el texto final que se diseñó desde la asignatura de Didáctica de la Lengua y la Literatura es una narración que integra los objetos seleccionados por los alumnos desde el ámbito de conocimiento de las ciencias sociales; mientras que desde la asignatura de enseñanza y aprendizaje de las matemáticas se investigan los conceptos numéricos y los patrones que se visibilizan mediante la construcción de la línea del tiempo. Esta narrativa multimodal final, consistía en un documento que podía incluir imágenes, sonidos, texto, vídeo o cualquier otra forma de expresión, y que debía exponerse de forma oral al resto del grupo. 
A lo largo del semestre, la docente de cada asignatura disponía de dos franjas horarias a la semana de dos horas cada una, durante ocho semanas. En una se trabajaba con la mitad del grupo (23 alumnos), mientras que la otra discurría con todos los alumnos. Así pues, cada profesora pudo adaptar y planificar su asignatura teniendo en cuenta esta flexibilidad organizativa, permitiendo desarrollar los contenidos y los aspectos didácticos y metodológicos propios de la materia. En un inicio, las docentes planificaron sesiones conjuntas con los alumnos para presentar y desarrollar el proyecto. La irrupción de la COVID-19 modificó esta idea inicial. A partir de este momento, en una de las dos franjas de cada asignatura, se llevaron a cabo tutorías virtuales con la participación de las docentes y los distintos grupos a partir de las dudas, sugerencias y de las propuestas de los estudiantes. En las últimas sesiones cada grupo expuso virtualmente su narración multimodal al resto de los compañeros.

La Figura 1 esquematiza la organización de la experiencia.

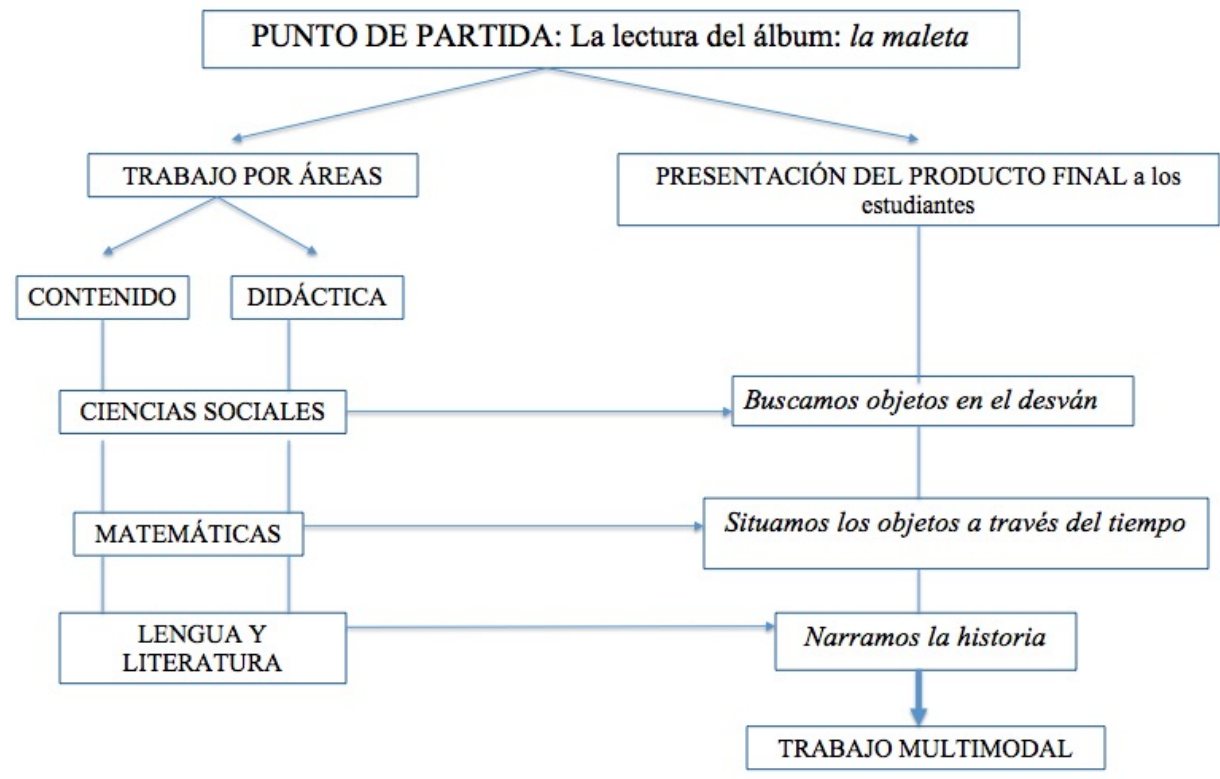

Figura 1. Organización de la experiencia Nuestra maleta.

\subsection{Objetivos, contenidos y competencias de Nuestra maleta}

Las características concretas de esta experiencia determinan que en la planificación del conjunto de competencias, objetivos y contenidos haya algunos que sean propiamente de cada asignatura, mientras que otros se compartan de manera transversal por las tres.

Con la intención de facilitar la lectura y comprensión de las competencias, objetivos y contenidos del proyecto, se exponen en la Tabla 1. 


\begin{tabular}{|c|c|c|c|}
\hline Ámbito & OBJETIVOS & CONTENIDOS & COMPETENCIAS \\
\hline $\begin{array}{l}\text { Lengua y } \\
\text { literatura }\end{array}$ & $\begin{array}{l}\text { Tener conciencia del valor } \\
\text { de la lectura de textos como } \\
\text { potencial para la escritura } \\
\text { ("leer para escribir"). } \\
\text {. Profundizar en el proceso } \\
\text { de escritura de un texto } \\
\text { narrativo y aplicar las bases } \\
\text { teóricas a la práctica } \\
\text { concreta. } \\
\text {. Utilizar diversos lenguajes } \\
\text { (trabajo multimodal) para } \\
\text { comunicar de forma } \\
\text { creativa la narrativa de cada } \\
\text { grupo. } \\
\text {. Reflexionar sobre la } \\
\text { transferencia de este tipo de } \\
\text { experiencias en el aula de } \\
\text { Educación Primaria. }\end{array}$ & $\begin{array}{l}\text {. La oralidad como elemento } \\
\text { fundamental en la creación y } \\
\text { expresión de las narrativas. } \\
\text {. Las estrategias de lectura y la } \\
\text { mediación del docente. } \\
\text {. El género narrativo. } \\
\text {. El proceso de creación de un } \\
\text { texto: (planificación, redacción } \\
\text { y revisión). } \\
\text {. La corrección lingüística. } \\
\text {. Las bases didácticas de la } \\
\text { construcción del texto narrativo. }\end{array}$ & $\begin{array}{l}\text { Expresarse con rigor lingǘ́stico } \\
\text { oralmente, por escrito y de forma } \\
\text { audiovisual. } \\
\text {. Profundizar sobre el proceso de } \\
\text { redacción del texto narrativo y } \\
\text { aplicarlo en el proyecto. } \\
\text {. Relacionar teoría y práctica con } \\
\text { la realidad del aula y de los } \\
\text { centros escolares. }\end{array}$ \\
\hline $\begin{array}{l}\text { Ciencias } \\
\text { sociales }\end{array}$ & $\begin{array}{l}\text { Relacionar el pasado con } \\
\text { la actualidad. } \\
\text { Plantear y resolver } \\
\text { problemas asociados con } \\
\text { las ciencias sociales en la } \\
\text { vida cotidiana. } \\
\text {. Llevar la historia y la } \\
\text { actualidad al aula }\end{array}$ & $\begin{array}{l}\text {. La actualidad en el aula } \\
\text {. El currículum del área de } \\
\text { Conocimiento del Medio: } \\
\text { selección de contenidos (los } \\
\text { relacionados con los objetos) } \\
\text {. Características del } \\
\text { conocimiento histórico, } \\
\text { geográfico, artístico y } \\
\text { económico }\end{array}$ & $\begin{array}{l}\text { Comprender la complejidad } \\
\text { social, la realidad cultural, } \\
\text { económica y política, y entenderlo } \\
\text { como un todo. } \\
\text {. Relacionar la realidad social, } \\
\text { económica, política y cultural con } \\
\text { el conocimiento científico. } \\
\text {. Profundizar en el desarrollo de } \\
\text { la competencia social y } \\
\text { ciudadana. }\end{array}$ \\
\hline Matemáticas & $\begin{array}{l}\text { Mostrar (los alumnos) } \\
\text { capacidad para usar las } \\
\text { matemáticas en diferentes } \\
\text { contextos o situaciones, } \\
\text { reflexionando acerca del } \\
\text { papel y el aporte de las } \\
\text { matemáticas en cada una de } \\
\text { ellas. } \\
\text {. Comunicar el pensamiento } \\
\text { matemático con coherencia } \\
\text { y claridad usando el } \\
\text { lenguaje matemático con } \\
\text { precisión. } \\
\text {. Reflexionar sobre el } \\
\text { potencial de las tareas, } \\
\text { como la línea del tiempo, } \\
\text { en el aula de Educación } \\
\text { Primaria. }\end{array}$ & $\begin{array}{l}\text {. La comprensión de los } \\
\text { números, de sus } \\
\text { representaciones y de las } \\
\text { relaciones entre ellos. } \\
\text {. El papel de los modelos } \\
\text { matemáticos para representar } \\
\text { y comprender las relaciones } \\
\text { cuantitativas. } \\
\text {. La comprensión de } \\
\text { patrones, estructuras y } \\
\text { relaciones; la utilización } \\
\text { de modelos matemáticos. }\end{array}$ & $\begin{array}{l}\text {. Adquirir competencias } \\
\text { matemáticas básicas de } \\
\text { numeración, cálculo, patrones, } \\
\text { entre otras. } \\
\text {. Plantear y resolver } \\
\text { situaciones de la vida } \\
\text { cotidiana. } \\
\text {. Valorar la relación entre las } \\
\text { matemáticas y otras } \\
\text { disciplinas; y el papel de las } \\
\text { matemáticas para comprender } \\
\text { el entorno social. }\end{array}$ \\
\hline $\begin{array}{l}\text { Trabajo } \\
\text { conjunto de } \\
\text { las tres } \\
\text { disciplinas }\end{array}$ & $\begin{array}{l}\text { Trabajar y reflexionar } \\
\text { sobre el trabajo } \\
\text { interdisciplinario en el aula. } \\
\text {. Mostrar creatividad en la } \\
\text { elaboración de la narrativa } \\
\text { multimodal. } \\
\text { - Relacionar distintas } \\
\text { disciplinas para elaborar } \\
\text { propuestas } \\
\text { interdisciplinares en la } \\
\text { educación. }\end{array}$ & $\begin{array}{l}\text {. El trabajo colaborativo } \\
\text { pequeño grupo. } \\
\text {. El uso de las TIC en la } \\
\text { producción de textos. } \\
\text {. Comprensión y elaboración de } \\
\text { nuevos conocimientos a partir } \\
\text { de los contenidos de cada } \\
\text { asignatura }\end{array}$ & $\begin{array}{l}\text {-Diseñar, planificar, desarrollar y } \\
\text { evaluar situaciones educativas } \\
\text { vinculadas a las tres disciplinas. } \\
\text {-Adquirir habilidades y } \\
\text { conocimientos que ayuden a } \\
\text { reflexionar sobre el hecho } \\
\text { educativo, sobre uno mismo, el } \\
\text { propio aprendizaje y el rol de } \\
\text { educador. } \\
\text {-Saber comunicarse a través de } \\
\text { los distintos lenguajes del } \\
\text { conocimiento científico. } \\
\text {-Analizar y construir el } \\
\text { conocimiento epistemológico de } \\
\text { aquellas disciplinas que } \\
\text { confluyen en la educación de los } \\
\text { niños de primaria. }\end{array}$ \\
\hline
\end{tabular}

Tabla 1. Ámbitos, objetivos, contenidos y competencias de la experiencia Nuestra maleta. 


\section{Desarrollo de la experiencia}

En este apartado se expone y explica de forma concreta y detallada cada una de las acciones realizadas en el transcurso de la experiencia. En los tres primeros apartados se describen las acciones desarrolladas desde cada asignatura que dibujan la propuesta. En el último se explicita la producción que integra todas las disciplinas.

\subsection{Punto de partida: lectura del álbum "La Maleta". Didáctica de la Lengua y la Literatura.}

La experiencia se inicia desde la asignatura de Didáctica de la Lengua y la Literatura a partir de la lectura en voz alta del álbum ilustrado titulado La maleta de Núria Parera. La narración es una ristra de relatos, que no pasan de diez líneas, donde se explican numerosos episodios históricos acaecidos durante el siglo XX de manera cronológica. La autora utiliza una maleta como objeto y personaje que va pasando de mano en mano y que se convierte en el testigo principal de los acontecimientos que se suceden en este recorrido histórico. El relato empieza en una pequeña localidad del Pirineo; desde allí la maleta recorre distintos escenarios geográficos, culturales, emocionales y cronológicos de diversos continentes. El álbum finaliza en Lesbos el año 2012.

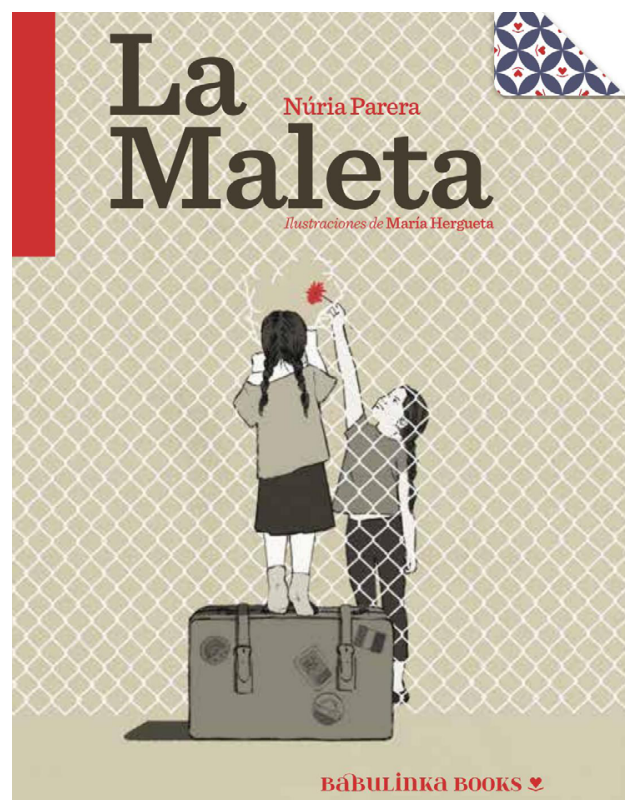

Figura 2. Parera, N. (2018). La maleta. Babulinka Libros.

La lectura de La maleta comporta el disfrute de una buena lectura en voz alta pero también constituye el punto de partida de la experiencia que conecta las tres asignaturas.

Antes, durante y después de la lectura se plantea a los alumnos un conjunto de actividades de comprensión de la historia que inciden en los distintos niveles de lectura: la lectura literal, la inferencial o profunda, así como también en la lectura crítica (Cassany, 2018). En estas actividades se pretende que pongan en juego distintas estrategias de comprensión lectora: formular hipótesis, descubrir sus conocimientos previos, realizar conexiones, formular(se) preguntas, clarificar y visualizar conceptos, inferir, buscar la idea principal, resumir, buscar el propósito de la autora, etc. 
Después de la lectura y de la comprensión del texto se explica a los alumnos el proyecto que se plantea desde las tres asignaturas y que tiene como producto final la realización de una narrativa multimodal.

\subsection{Buscamos objetos en el desván. Conocimiento del Medio Cultural y Social.}

Desde la asignatura de Ciencias Sociales, el trabajo se introduce a los alumnos a partir de un soporte digital que muestra los objetos que supuestamente la profesora ha encontrado en el desván de casa de sus abuelos; objetos que los estudiantes del grado de Educación Primaria también pueden encontrar: elementos antiguos como monedas, documentos, fotografías, armas, objetos personales (de aseo, del hogar, de vestuario,...), cromos, mapas, anuncios, películas antiguas, recortes de prensa, carteles, fotos y novelas de antaño, entre otros.

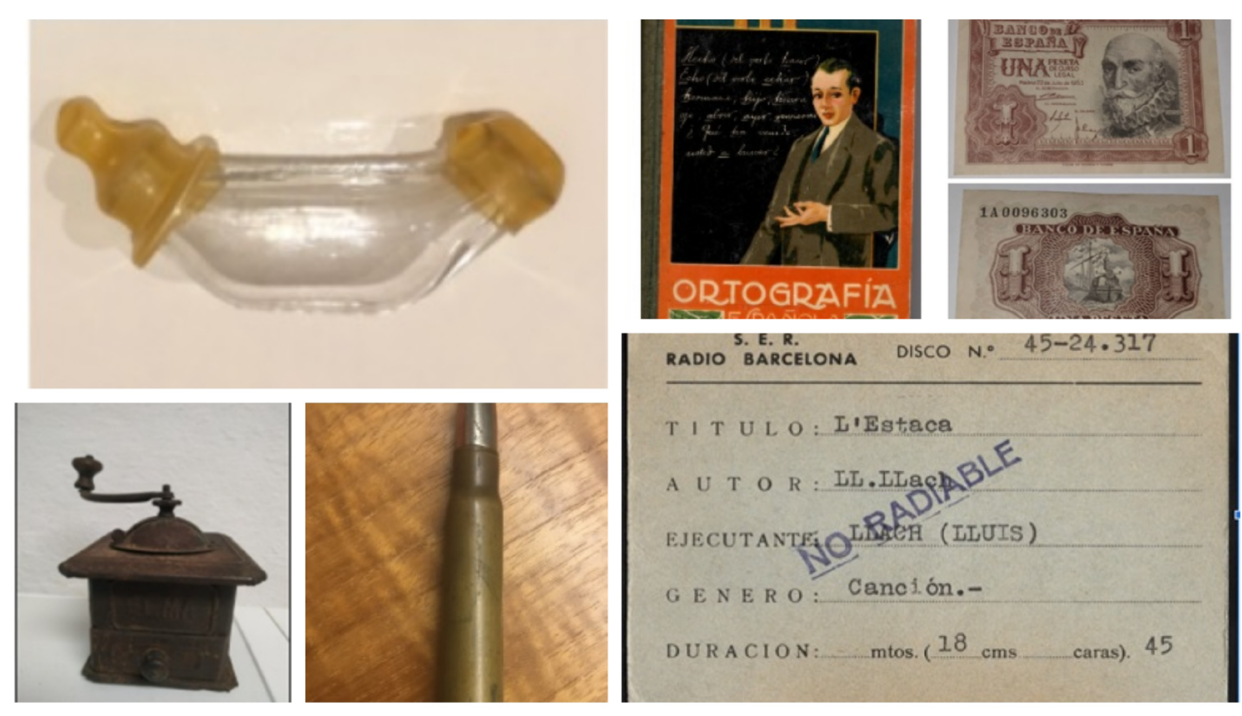

Figura 3. Ejemplos de objetos encontrados en el desván.

Teniendo en cuenta que los alumnos están organizados por grupos de trabajo, se pide a cada integrante que aporte dos o tres objetos del desván de sus abuelos. Cada grupo debe escoger tres objetos del total que reúnan algunas características comunes entre ellos, ya sea por ser de la misma época, por ser de épocas cronológicamente consecutivas o por tener relación temática, de lugar, etc. Cada grupo selecciona, a partir del criterio que considere más conveniente, los objetos encontrados. Posteriormente deben identificar el contexto histórico de los objetos seleccionados e investigar su historia para poder explicar el periodo histórico que han escogido, utilizando los objetos como fuentes primarias.

\subsection{Situamos los objetos a través del tiempo. Didáctica de las Matemáticas}

Los objetos que cada grupo de alumnos escoge en la asignatura de Sociales, así como las diferentes referencias temporales surgidas, son el punto de partida para elaborar una línea del tiempo que permite a cada grupo reflexionar acerca de la numeración, los patrones y la medida. 
Por grupos de trabajo documentan y reflexionan sobre el proceso de construcción de la línea del tiempo; desde los aspectos técnicos (material utilizado, longitud de un año, de una década, de un siglo), hasta los aspectos del uso didáctico de la línea: cómo comparar periodos de tiempo, cómo entender la relación entre las diferentes medidas de tiempo (año, década...), cómo saber "cuánto tiempo ha transcurrido desde que..." o "cuánto tiempo ha transcurrido desde que pasó... hasta que pasó...", etc. Así, con esta actividad cada grupo conecta las experiencias vitales que aparecen en su línea del tiempo con tareas matemáticas útiles que les permiten introducir conceptos matemáticos importantes, y que a su vez implican y retan intelectualmente a los alumnos (National Council of Teachers of Mathematics, 2000).

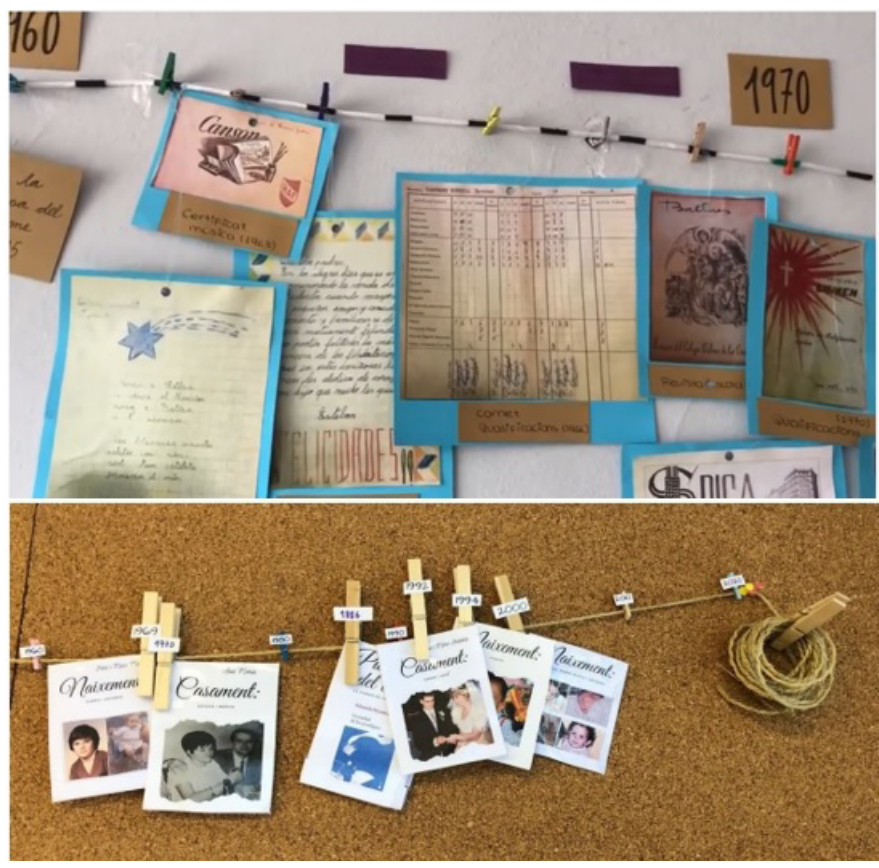

Figura 4. Ejemplos de líneas del tiempo.

Las líneas del tiempo de los diferentes grupos resultan distintas y variadas, ya que recogen eventos, momentos o periodos temporales propios de cada grupo, de los objetos elegidos y/o de la temática relacionada con dichos objetos. Finalmente, para que la línea adquiera sentido para ellos, incluyen algún elemento o fecha de conexión con la vida del grupo, individual o colectivamente.

La elaboración de la línea del tiempo no solo enlaza con el trabajo realizado en la asignatura de actualización curricular y científica de las Ciencias Sociales, sino que constituye el primer paso para analizar y reflexionar acerca de una experiencia de aula similar llevada a cabo por Forrellad, Rigol y Gallego (2003), con alumnos de ciclo inicial. Se trata, pues, de una propuesta doble. Inicialmente, los alumnos elaboran su propia línea del tiempo a la vez que descubren sus posibilidades para trabajar aspectos de numeración, de patrones y de medida. Posteriormente, analizan una experiencia similar desarrollada en el ciclo inicial de primaria donde se pone de manifiesto el papel de las matemáticas para construir nuevos conocimientos. 


\subsection{Narramos la historia. Didáctica de la Lengua y la Literatura}

A lo largo de las sesiones en que los alumnos, desde las áreas de Ciencias Sociales y Matemáticas, avanzan en la construcción de los elementos que constituirán la base de su narración, desde la Didáctica de la Lengua y la Literatura se analiza cuáles son los elementos fundamentales del texto narrativo, así como los planteamientos didácticos necesarios para trabajar con los alumnos de primaria. Reflexionan sobre cómo trabajar los tres momentos de la construcción de un texto: la planificación, la redacción y la revisión.

En la planificación los alumnos deben tener en cuenta cuál es su contexto geográfico y social, es decir, el dónde - ya trabajado desde las Ciencias Sociales- pero también el cuándo; es decir, el tiempo en la narración que ya se había considerado desde el área de matemáticas.

Una vez contextualizada la narración, cada grupo de alumnos determina los personajes: los principales y secundarios, pero también concretan si aparecerá en su historia algún personaje antagonista. En muchas de las narraciones que crean los estudiantes los objetos seleccionados previamente se pueden considerar como auténticos personajes de la narración, y a menudo, comparten espacio y protagonismo con los personajes humanos que representan distintas generaciones y clases sociales.

Figura 5. Ejemplo de ambientación de personajes con objetos.

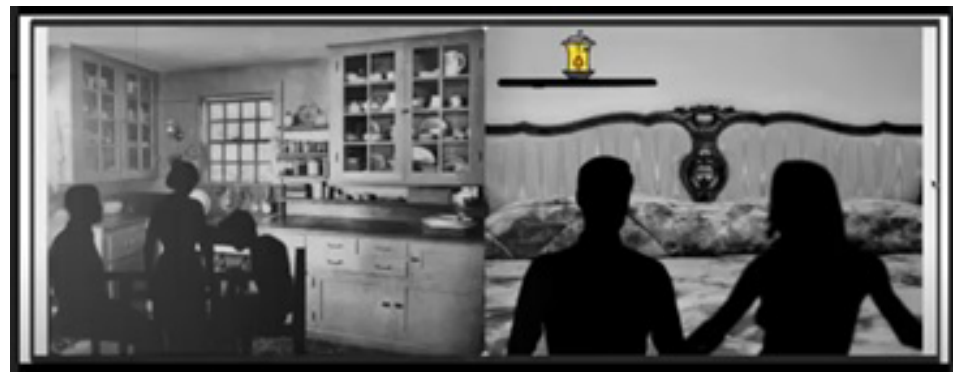

En este momento del proceso de planificación, los estudiantes ya están en condiciones para pensar la trama y la acción de la narración. Este trabajo requiere tiempo y aportaciones por parte de todos los miembros del grupo. Ellos, en un inicio de la experiencia, habían escuchado una narración, la historia de La maleta, que no solo es el punto de partida de la experiencia y una actividad gratificante, -la escucha de una historia en voz alta-, sino que desempeñaba también una función didáctica: leer una narración para aprender a escribir una historia.

Una vez decidida la trama de su narración, los diferentes grupos inician el trabajo de la redacción del texto. El inicio de la pandemia obligó a que el trabajo colaborativo entre ellos, pero también las tutorías con las profesoras se llevaran a cabo de manera virtual. Aquí, las sesiones se organizaron tanto de forma sincrónica como diacrónica en formato de videoconferencia y la tecnología ayudó enormemente en este proceso.

La producción de los diversos textos culmina con la estrategia de la revisión de éstos por parte de los alumnos (Cassany 2007). Para ello usan diferentes herramientas de consulta (diccionarios, correctores digitales y en papel, libros de estilo, etc.) que les permiten revisar y mejorar su texto (D'Aquino, 2016). 


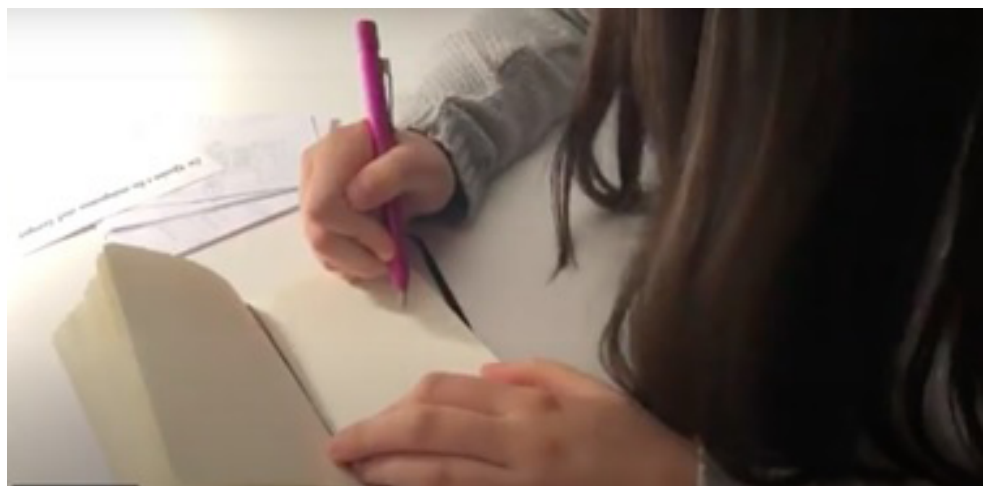

Figura 6. Proceso de creación y redacción de la narrativa.

\subsection{Trabajo multimodal: Narrativa. Una propuesta conjunta.}

Como ya se ha dicho anteriormente, el producto final, en un inicio, consistía en una exposición oral presencial conjunta para las tres materias. A partir de la cronología establecida mediante la línea del tiempo, cada grupo presentaba a sus compañeros los objetos encontrados mediante una narrativa corta elaborada por ellos. Se tomó como ejemplo la actividad de lectura en voz alta del álbum ilustrado titulado La maleta de Núria Parera (2018).

Cuando el 13 de marzo de 2020 se produce el confinamiento, se replantea la exposición inicial adaptándola a un formato virtual multimodal. La propuesta final seguía siendo la elaboración de una historia o narración inventada por cada grupo, pero se modifica el soporte de presentación. Se eligió el formato de vídeo ya que permitía superar la incertidumbre del confinamiento sin modificar, de manera sustancial, la actividad que se planteó inicialmente a los alumnos. Tener la narración en un video permitía corregirlo y presentarlo virtualmente si seguíamos confinados hasta final de curso, y en el caso de no estarlo se podían ver los vídeos en clase y completar la presentación con la explicación de los objetos y la línea del tiempo.

El vídeo final tenía como objetivo ser el reflejo de un trabajo interdisciplinar realizado por cada grupo, que en ningún caso fuera compartimentado. A su vez, debía ser multimodal pudiendo utilizar diversas herramientas (vídeo, videoScribe, etc.) y formas de comunicación (grabación, imágenes fijas, vídeos, música, etc.) Se proponía que la duración del vídeo fuera de máximo cinco minutos y que, en la grabación, todos los miembros del grupo narraran.

La realidad de la pandemia provocada por la COVID-19 fue muy dura para los mayores, por lo que, a pocas semanas de la presentación del trabajo, y teniendo en cuenta que los objetos elegidos por los alumnos estaban muy relacionados con sus abuelos, consideramos que era oportuno hacerles un pequeño homenaje. Por ello les propusimos que preparasen un segundo vídeo, muy corto (máximo 30 segundos) dedicado a sus abuelos. Cada grupo tenía libertad absoluta para homenajearlos. La Facultad elaboró posteriormente, con el consentimiento de los alumnos, un vídeo con parte de los homenajes elaborados y que puede verse en las redes sociales: https://www.instagram.com/p/CAXhOmcqJnK/?igshid=1ktzygtoog1yq. 


\section{Evaluación de "Nuestra Maleta"}

Por lo que se refiere al proceso previo a la creación de la narrativa, la evaluación de las distintas evidencias por materias sufrió algún cambio respecto al planteamiento inicial al tenerse que realizar de manera virtual. La profesora de cada materia entregó a los distintos grupos un pequeño informe del trabajo, con la evaluación numérica y formativa, acompañada de comentarios que explicitaban los puntos fuertes y los aspectos a mejorar. Estos se elaboraron a partir de rúbricas ad hoc para la experiencia. Los grupos que lo solicitaron tuvieron la posibilidad de realizar tutorías virtuales con la profesora para aclarar dudas.

En el caso de la narrativa final, todas las profesoras visualizaron y valoraron el vídeo juntamente con los alumnos, focalizando la atención en los siguientes aspectos:

- $\quad$ Estructura de la presentación

- Contextualización del trabajo

- $\quad$ Expresión oral (dicción, riqueza de vocabulario, entre otros)

- Coherencia de la presentación

- Incorporación de los elementos específicos de ciencias sociales, matemáticas y lengua.

- $\quad$ Concreción, claridad e hilo de la narración

La evaluación final de la narrativa tuvo una valoración cuantitativa, compartida por las profesoras y que incidía en las tres asignaturas, y otra formativa, en formato de informe con los puntos fuertes y los aspectos a mejorar.

\section{Valoración de la experiencia por parte de los alumnos}

En este apartado se comenta la valoración que hicieron los alumnos, tanto del proceso seguido como de su producto final. Estas evaluaciones son de gran ayuda para mejorar y avanzar en actividades interdisciplinares futuras.

\subsection{Valoración de la narrativa final por parte de los alumnos}

Los alumnos valoraron la posibilidad de poder vivir una experiencia en primera persona, lo que les permitió descubrir las dificultades y los retos que deberán tener en cuenta cuando realicen una propuesta similar con los alumnos de primaria. Otro aspecto bien valorado fue la importancia de trabajar a partir de objetos reales y familiares porque les permitió establecer el vínculo y el conocimiento de la historia familiar, a la vez que implicó una actividad significativa para los alumnos.

Uno de los grupos remarcó el valor que tuvo para ellos el hallazgo de una cinta en audio de una de sus abuelas; de esta manera no solo tenían su recuerdo físico a partir de las fotografías, sino también de algo tan característico como es el registro oral. Consideraron que es importante transmitir el valor y significado de las fuentes primarias a sus futuros alumnos.

Los distintos grupos también valoraron positivamente el hecho de tener que poner en juego distintas habilidades para llevar a cabo el proyecto (búsqueda de información, habilidades propias de 
redacción, de aspectos matemáticos, pero también tecnológicas y creativas). También remarcaron que les permitió conocer habilidades de otros compañeros que hasta ese momento desconocían.

Por lo que refiere a los retos propios del trabajo que los alumnos tuvieron que superar, destacaron, especialmente, la complejidad en la elaboración del texto. Algunos grupos coincidieron en la dificultad de elaborar una narrativa que no fuera excesivamente descriptiva y que invitara al lector a una lectura atenta.

\subsection{Valoración de la experiencia "Nuestra maleta" por parte de los alumnos}

Los comentarios de los alumnos acerca de la experiencia "Nuestra maleta" que incluye las tres disciplinas se pueden agrupar en tres aspectos: los más valorados, los retos presentados y que tuvieron que resolver, y las propuestas de mejora.

Los alumnos destacaron el acompañamiento del profesorado, no solamente en la resolución de cuestiones referidas al trabajo, sino también en las propuestas de retos para mejorar la calidad de su proyecto.

Los alumnos también comentaron que a menudo muestran poco interés por alguna de estas tres materias porque las asocian a un cúmulo de datos sin relación con la vida o a cálculos sin sentido. Sin embargo, este trabajo les permitió llegar al conocimiento conceptual a partir de una experiencia familiar y les ha reconciliado con el conocimiento de estas materias. Para los alumnos fue también una manera de dar las gracias a sus abuelos, porque les ha permitido contar su historia. En la situación de pandemia que nos ha tocado vivir, terminar el trabajo con un homenaje a los abuelos lo han vivido como una experiencia emocionante.

Referente a los retos y dificultades que tuvieron que resolver, destacaron especialmente las derivadas de la situación de la pandemia: no poderse reunir físicamente; un trabajo siempre a distancia con todas las dificultades que eso conlleva en la realización material y física de la narrativa, etc. Cabe destacar que consideraron que estas dificultades les obligaron a repensar y buscar soluciones imaginativas y coherentes (pensar otros formatos de presentación de las narrativas, otras maneras de hacer el trabajo conjunto, etc.)

Como propuesta de mejora, los alumnos consideraron que hubiera sido útil que la presentación de la propuesta "Nuestra maleta", en lugar de realizarse paulatinamente siguiendo un planteamiento didáctico, se hubiera presentado globalmente desde el inicio del semestre para poderse organizar mejor.

\section{Reflexión final y prospectiva}

Un trabajo como el planteado nace de un deseo de ofrecer a los alumnos propuestas de aula ricas en contenido tanto conceptual como didáctico. Poder realizar la experiencia en primera persona, tomando elementos cotidianos y próximos ha permitido a los alumnos del grado de Educación Primaria vivir una propuesta similar a la que pueden presentar a sus futuros alumnos en el aula. Por consiguiente, los retos que han superado o las dificultades vividas les permitirán afrontar experiencias similares conociendo los desafíos, las dificultades y los aprendizajes que pueden ofrecer propuestas similares. 
Los alumnos han conectado distintas disciplinas que tal vez a priori podían parecerles realidades lejanas. Han hilvanado relaciones y han descubierto las que se establecen en los trabajos de sus compañeros.

El trabajo conjunto ha significado para las docentes un enriquecimiento cognitivo y un replanteamiento metodológico. La propia metodología seguida por las profesoras puede ser para los alumnos un ejemplo de team teaching que permite la conexión entre disciplinas a partir de la realidad y por lo tanto, un aprendizaje construido desde diferentes perspectivas

Nos sentimos satisfechas con las narrativas finales de los alumnos y tomamos en consideración sus valoraciones y sus propuestas de mejora. Pero sobre todo nos sentimos animadas para mejorar la experiencia y para seguir planteando propuestas estimulantes en nuestras aulas.

En el futuro pensamos iniciar un nuevo proyecto, recogiendo las inquietudes de los alumnos, manteniendo un planteamiento interdisciplinar, pero buscando nuevos puntos de inicio y productos finales.

\section{Referencias}

Andonegui, M. (2004). Interdisciplinariedad y educación matemática en las dos primeras etapas de la educación básica. Educere, 8(26), 301-308.

http://erevistas.saber.ula.ve/index.php/educere/article/view/12010

Beninghof, A. M. (2012). Co-teaching that works: Structures and strategies for maximizing student learning. Jossey-Bass.

Cassany, D. (2007). Afilar el lapicero. Editorial Anagrama.

Cassany, D. (2018). Laboratorio lector para entender la lectura. Editorial Anagrama.

Cook, L. (2004). Co-teaching: principles, practices, and pragmatics. http://files.eric.ed.gov/fulltext/ED486454.pdf

Cook, L. y Friend, M. (1995). Co-teaching: Guidelines for creating effective practices. Focus on Exceptional Children, 28(3), 1-16. https://doi.org/10.17161/foec.v28i3.6852

Conderman, G. y Hedin, L. (2012). Purposeful assessment practices for co-teachers. Teaching Exceptional Children, 44(4), 19-27. https://doi.org/10.1177/004005991204400402

D’Aquino, A. (2016). Dels errors se n'aprèn. La correcció com a instrument didàctic. Articles de Didàctica de la Llengua i la Literatura, 68, 7-13. http://hdl.handle.net/11162/115806

Dugan, K. y Letterman, M. (2008). Student appraisals of collaborative teaching. College Teaching, 56(1), 11-15. https://doi.org/10.3200/CTCH.56.1.11-16

Folch, C., Capdevila, R. y Prat, M. (2019). Percepción del Profesorado sobre una Experiencia Multidisciplinar: Arte y Ciencias en un Grado de Educación. Revista Digital de Investigación en Docencia Universitaria, 13(1), 38-56. https://doi.org/10.19083/ridu.2019.743

Follari, R. (2013). Acerca de la interdisciplina: posibilidades y límites. Interdisciplina I, 1, 111-130. http://dx.doi.org/10.22201/ceiich.24485705e.2013.1.46517

Forrellad, H., Rigol, A. y Gallego, C. (2003). La máquina del tiempo. Revista Kikirikí Cooperación Educativa, 58, 73-82. https://bit.ly/2VIgzro

Friend, M. (2008). Co-teaching a simple solution That isn't simple after all. Journal of Curriculum and Instruction, 2(2), 9-19. https://doi.org/10.3776/joci.2008.v2n2p9-19

Lenoir, Y. (2013). Interdisciplinariedad en educación: una síntesis de sus especificidades y actualización. Interdisciplina I, 1, 51-86. http://dx.doi.org/10.22201/ceiich.24485705e.2013.1.46514 
National Council of Teachers of Mathematics (NCTM) (2000). Principios y estándares para la educación matemática. SAEM Thales.

Nicolescu, B. (1998). The transdisciplinary evolution of the university, condition for sustainable development CIRET. Butlletin Interactif du Centre International de Recherches et Études transdisciplinaires,12. http://ciret-transdisciplinarity.org/bulletin/b12c8.php

Parera, N. (2018). La maleta. Babulinka Libros

Suárez-Díaz, G. (2016). Co-enseñanza: concepciones y prácticas en profesores de una Facultad de Educación en Perú. Revista Electrónica de Investigación Educativa, 18(1), 166-182.

http://redie.uabc.mx/redie/article/view/786 